Research Article

Mohamed Jleli*

\title{
Instantaneous blow-up of solutions to the Cauchy problem for the fractional Khokhlov-Zabolotskaya equation
}

https://doi.org/10.1515/math-2020-0089

received September 5, 2020; accepted September 27, 2020

Abstract: In this paper, we consider the Cauchy problem for a second-order nonlinear equation with mixed fractional derivatives related to the fractional Khokhlov-Zabolotskaya equation. We prove the nonexistence of a classical local in time solution. The obtained instantaneous blow-up result is proved via the nonlinear capacity method.

Keywords: nonlinear capacity, blow-up, fractional differential equations

MSC 2020: 26A33

\section{Introduction}

The study of the blow-up phenomena for many initial-boundary value problems that arise in particular in the theory of ion sound waves in plasma [1], waves in semiconductors [2] and electric oscillations [3] was detailed in many studies with the aim to obtain global or local unsolvability results by using the method of test functions.

The Khokhlov-Zabolotskaya equation arose in the theory of nonlinear underwater acoustics. This equation has the following form (see [4]):

$$
\partial_{t} \partial_{x} u(t, w)-\frac{c_{0}}{2} \Delta_{(y, z)} u(t, w)=\frac{\varepsilon}{2 c_{0}^{2}} \partial_{t}^{2} u^{2}(t, w),
$$

for $t>0, w \in \mathbb{R}^{3}, c_{0}$ is the equilibrium speed of sound and $\varepsilon$ is a nonlinear acoustic parameter. Here

$$
\Delta_{(y, z)} u(t, w):=\left(\partial_{y}^{2}+\partial_{z}^{2}\right) u(t, w), \quad \text { for } w=(x, y, z) \in \mathbb{R}^{3} .
$$

Recently, in [5], the Cauchy problem for the aforementioned second-order nonlinear equation with mixed derivatives with the initial data

$$
u(0, w)=u_{0}(w) \quad \text { and } \quad u_{t}(0, w)=u_{1}(w), \quad \text { for } w \in \mathbb{R}^{3},
$$

was studied. In particular, the blow-up of its classical local-in-time solution is proved.

Based on the nonlinear capacity method developed in [6], in this paper, we obtain an instantaneous blow-up result for the fractional version of the Khokhlov-Zabolotskaya equation. More precisely, let $T>0$ be fixed, $\alpha \in(0,1)$ and $Q_{T}=[0, T] \times \mathbb{R}^{3}$. First, we consider the problem

$$
\partial_{t}^{\alpha} \partial_{x} u(t, w)-\Delta_{(y, z)} u(t, w)=\frac{1}{2} \partial_{t}^{\alpha+1} u^{2}(t, w), \quad(t, w) \in Q_{T},
$$

\footnotetext{
* Corresponding author: Mohamed Jleli, Department of Mathematics, College of Science, King Saud University, P.0. Box 2455, Riyadh, 11451, Saudi Arabia, e-mail: jleli@ksu.edu.sa 
under the initial data

$$
u(0, w)=u_{0}(w) \text { and } u_{t}(0, w)=u_{1}(w), \quad \text { for } w \in \mathbb{R}^{3},
$$

where $\partial_{t}^{\alpha} u$ is the left-sided Liouville-Caputo derivative of order $\alpha$ of the function $u$.

Then, for $\alpha, \beta \in(0,1)$ we will be interested in the system

$$
\left\{\begin{array}{l}
\partial_{t}^{\alpha} \partial_{x} u(t, w)-\Delta_{(y, z)} u(t, w)=\frac{1}{2} \partial_{t}^{\alpha+1} v^{2}(t, w) \text { in } Q_{T}, \\
\partial_{t}^{\beta} \partial_{x} v(t, w)-\Delta_{(y, z)} v(t, w)=\frac{1}{2} \partial_{t}^{\beta+1} u^{2}(t, w) \text { in } Q_{T},
\end{array}\right.
$$

under the initial data

$$
u(0, w)=u_{0}(w), \quad u_{t}(0, w)=u_{1}(w), \quad v(0, w)=v_{0}(w) \quad \text { and } \quad v_{t}(0, w)=v_{1}(w) \quad \text { for } w \in \mathbb{R}^{3} .
$$

\section{Preliminaries}

Recall that, for $\alpha \in(0,1)$ and $\Psi \in C^{2}[0, T]$, the left-sided and right-sided fractional derivatives of orders $\alpha$ and $\alpha+1$ of the function $\Psi$ are given by

$$
\partial_{t}^{\alpha} \Psi(t)=\mathcal{I}_{0 \mid t}^{1-\alpha}\left(\Psi_{t}\right)(t) \quad \text { and } \quad \partial_{t}^{1+\alpha} \Psi(t)=\mathcal{I}_{0 \mid t}^{1-\alpha}\left(\Psi_{t t}\right)(t),
$$

where

$$
I_{0 \mid t}^{\alpha}(\Psi)(t)=\frac{1}{\Gamma(\alpha)} \int_{0}^{t}(t-s)^{\alpha-1} \Psi(s) \mathrm{d} s \quad \text { and } \quad I_{t \mid T}^{\alpha}(\Psi)(t)=\frac{1}{\Gamma(\alpha)} \int_{0}^{t}(s-t)^{\alpha-1} \Psi(s) \mathrm{d} s
$$

are the left-sided and right-sided fractional integrals of order $\alpha$. It is easy (see [7]) to see that for all $\alpha \in(0,1)$ and $\Psi, \Phi \in C[0, T]$, we have

$$
\int_{0}^{T}\left(\mathcal{I}_{0 \mid t}^{\alpha} \Psi\right)(t) \Phi(t) \mathrm{d} t=\int_{0}^{T} \Psi(t)\left(\mathcal{I}_{t \mid T}^{\alpha} \Phi\right)(t) \mathrm{d} t .
$$

For $T>0, \alpha \in(0,1)$ and $R \gg 1$, we define

$$
[0, T] \ni t \mapsto v(t):=\left(1-\frac{t}{T}\right)^{1+\alpha}, \quad \text { and } \quad \mathbb{R}^{3} \ni w \mapsto \mu(w):=\xi^{4}\left(\frac{x^{2}+R^{2}\left(y^{2}+z^{2}\right)}{R^{4}}\right),
$$

with $\xi \in C^{\infty}([0, \infty))$ be defined by

$$
\xi(r)= \begin{cases}1, & 0 \leq r \leq 1 \\ \searrow, & 1 \leq r \leq 2 \\ 0, & r \geq 2\end{cases}
$$

Also, we denote by $Q_{T, R}=[0, T] \times B_{R}$, where

$$
B_{R}=\left\{w \in \mathbb{R}^{3} ; \quad 1 \leq \frac{x^{2}+R^{2}\left(y^{2}+z^{2}\right)}{R^{4}} \leq 2\right\} .
$$

Using (6) and (8), it is easy to show that

Lemma 2.1. Let $\alpha \in(0,1)$ and $k \in\{0,1,2\}$. Then

$$
\left(\mathcal{I}_{t \mid T}^{1-\alpha} v\right)^{(k)}(t)=(-1)^{k} \frac{\Gamma(2+\alpha)}{\Gamma(3-k)} T^{-(1+\alpha)}(T-t)^{2-k}, \text { for all } t \in[0, T] .
$$


Lemma 2.2. For all $R>0$, there exists $c_{\xi}>0$ depending only on $\xi$ such that

$$
\int_{B_{R}} \frac{\left|\Delta_{(y, z)} \mu(w)\right|^{2}+\left|\partial_{x} \mu(w)\right|^{2}}{\mu(w)} \mathrm{d} w \leq c_{\xi}
$$

\section{Results}

Let $T>0$, be fixed, $\alpha \in(0,1)$ and $R \gg 1$. We define

$$
Q_{T} \ni(t, w) \mapsto \eta(t, w):=v(t) \mu(w),
$$

where the function $v$ and $\mu$ are given in (8).

We have the following result:

Theorem 3.1. Let $u_{0}$ and $u_{1}$ be two functions defined on $\mathbb{R}^{3}$ such that $u_{0} \in L^{2}\left(\mathbb{R}^{3}\right)$ and $u_{0} u_{1} \in L^{1}\left(\mathbb{R}^{3}\right)$. Then

- If $u_{0} \equiv 0$ a.e. and $u_{1} \neq \equiv$, then the instantaneous blow-up occurs for any solution to (1)-(2).

- If $u_{0} \neq \equiv 0$ and $\int_{\mathbb{R}^{3}} u_{0}(w) u_{1}(w) \mathrm{d} w<0$, then the time period existence $T_{\max }$ for any local solution to (1)-(2) satisfies

$$
T_{\max } \leq-\frac{\int_{\mathbb{R}^{3}} u_{0}(w) u_{1}(w) \mathrm{d} w}{\int_{\mathbb{R}^{3}} u_{0}^{2}(w) \mathrm{d} w}
$$

and for all $0<T<T_{\max }$, we have

$$
\int_{Q_{T}} u^{2}(t, w) \mathrm{d} t \mathrm{~d} w \leq \int_{\mathbb{R}^{3}}\left(T u_{0}^{2}(w)+T^{2} u_{0}(w) u_{1}(w)\right) \mathrm{d} w .
$$

Theorem 3.2. Let $u_{0}, v_{0}, u_{1}$ and $v_{1}$ be defined on $\mathbb{R}^{3}$ such that $u_{0}, v_{0} \in L^{2}\left(\mathbb{R}^{3}\right)$, and $u_{0} u_{1}, v_{0} v_{1} \in L^{1}\left(\mathbb{R}^{3}\right)$. Then

- If $u_{0} \equiv 0$ a.e. and $u_{1} \neq 0$ or $v_{0} \equiv 0$ a.e. and $v_{1} \neq 0$, then the instantaneous blow-up occurs for the solution of systems (3) and (4).

- If $u_{0} \neq \equiv 0, v_{0} \neq \equiv 0, \int_{\mathbb{R}^{3}} u_{0}(w) u_{1}(w) \mathrm{d} w<0$ and $\int_{\mathbb{R}^{3}} v_{0}(w) v_{1}(w) \mathrm{d} w<0$, then the period existence $T_{\max }$ for any local pair solution to (3) and (4) satisfies

$$
T_{\max } \leq-\max \left\{\frac{\int_{\mathbb{R}^{3}} u_{0} u_{1} \mathrm{~d} w}{\int_{\mathbb{R}^{3}} u_{0}^{2} \mathrm{~d} w}, \frac{\int_{\mathbb{R}^{3}} v_{0} v_{1} \mathrm{~d} w}{\int_{\mathbb{R}^{3}} v_{0}^{2} \mathrm{~d} w}\right\},
$$

and for all $0<T<T_{\max }$, we have

$$
\int_{Q_{T}} u^{2} \mathrm{~d} t \mathrm{~d} w \leq \int_{\mathbb{R}^{3}}\left(T u_{0}^{2}+T^{2} u_{0} u_{1}\right) \mathrm{d} w \text { and } \int_{Q_{T}} v^{2} \mathrm{~d} t \mathrm{~d} w \leq \int_{\mathbb{R}^{3}}\left(T v_{0}^{2}+T^{2} v_{0} v_{1}\right) \mathrm{d} w .
$$

Proof. Assume that there exists a solution $u$ to (1) and (2), defined on $Q_{T}$ for some $T>0$. Then multiplying (1) by $\eta$, we get

$$
\frac{1}{2} \int_{Q_{T}} \eta\left(\partial_{t}^{\alpha+1} u^{2}\right) \mathrm{d} t \mathrm{~d} w=\int_{Q_{T}} \eta\left(\partial_{t}^{\alpha} \partial_{x} u\right) \mathrm{d} t \mathrm{~d} w-\int_{Q_{T}} \eta\left(\Delta_{(y, z)} u\right) \mathrm{d} t \mathrm{~d} w
$$

Thanks to (7), have 


$$
\begin{aligned}
\int_{Q_{T}} \eta(t, w)\left(\partial_{t}^{\alpha} \partial_{x} u\right)(t, w) \mathrm{d} t \mathrm{~d} w & =\int_{0}^{T} v(t)\left(\int_{\mathbb{R}^{3}} \partial_{x}\left(\partial_{t}^{\alpha} u\right)(t, w) \mu(w) \mathrm{d} w\right) \mathrm{d} t \\
& =-\int_{0}^{T} v(t)\left(\int_{\mathbb{R}^{3}}\left(\partial_{t}^{\alpha} u\right)(t, w) \partial_{x} \mu(w) \mathrm{d} w\right) \mathrm{d} t \\
& =-\int_{B_{R}} \partial_{x} \mu(w)\left(\int_{0}^{T}\left(\mathcal{I}_{0 \mid t}^{1-\alpha} u_{t}\right)(t, w) v(t) \mathrm{d} t\right) \mathrm{d} w \\
& =-\int_{B_{R}} \partial_{x} \mu(w)\left(\int_{0}^{T} u_{t}(t, w)\left(I_{t \mid T}^{1-\alpha} v\right)(t) \mathrm{d} t\right) \mathrm{d} w \\
& =\int_{Q_{T, R}} \partial_{x} \mu(w)\left(\mathcal{I}_{t \mid T}^{1-\alpha} v\right)_{t}(t) \mathrm{d} t \mathrm{~d} w-\int_{B_{R}} \partial_{x} \mu(w)\left[u\left(I_{t \mid T}^{1-\alpha} v\right)_{t}(t)\right]_{t=0}^{t=T} \mathrm{~d} w .
\end{aligned}
$$

Therefore, from Lemma 2.1, we deduce that

$$
\int_{Q_{T}} \eta\left(\partial_{t}^{\alpha} \partial_{x} u\right) \mathrm{d} t \mathrm{~d} w=\frac{\Gamma(2+\alpha) T^{1-\alpha}}{2}\left(\int_{B_{R}} u_{0} \partial_{x} \mu \mathrm{d} w-2 T^{-2} \int_{Q_{T, R}} u \partial_{x} \mu(T-t) \mathrm{d} t \mathrm{~d} w\right) .
$$

Similarly, using (7) and Lemma 2.1, we obtain

$$
\begin{aligned}
\int_{Q_{T}} \eta\left(\partial_{t}^{\alpha+1} u^{2}\right) \mathrm{d} t \mathrm{~d} w & =\int_{\mathbb{R}^{3}} \mu(w)\left(\int_{0}^{T} v(t)\left(\partial_{t}^{1+\alpha} u^{2}\right)(t) \mathrm{d} t\right) \mathrm{d} w \\
& \left.=\int_{\mathbb{R}^{3}} \mu(w)\left(\int_{0}^{T}\left(I_{0 \mid t}^{1-\alpha} u^{2}\right)_{t t}\right)(t, w) v(t) \mathrm{d} t\right) \mathrm{d} w \\
& =\int_{\mathbb{R}^{3}} \mu(w)\left(\int_{0}^{T}\left(u^{2}\right)_{t t}(t, w)\left(I_{t \mid T}^{1-\alpha} v\right)(t) \mathrm{d} t\right) \mathrm{d} w \\
& =\int_{Q_{T}} u^{2} \mu(w)\left(I_{t \mid T}^{1-\alpha} v\right)_{t t}(t) \mathrm{d} t \mathrm{~d} w+\int_{\mathbb{R}^{3}} \mu(w)\left[\left(u^{2}\right)_{t}\left(I_{t \mid T}^{1-\alpha} v\right)-u^{2}\left(I_{t \mid T}^{1-\alpha} v\right)_{t}\right]_{t=0}^{t=T} \mathrm{~d} w \\
& =\Gamma(2+\alpha) T^{-(1+\alpha)}\left(\int_{Q_{T}} u^{2} \mu \mathrm{d} t \mathrm{~d} w-\int_{\mathbb{R}^{3}}\left(T u_{0}^{2}+T^{2} u_{0} u_{1}\right) \mu \mathrm{d} w\right) .
\end{aligned}
$$

On the other hand,

$$
\int_{Q_{T}} \eta(t, w)\left(\Delta_{(y, z)} u\right)(t, w) \mathrm{d} t \mathrm{~d} w=\int_{Q_{R, T}} u(t, w) v(t)\left(\Delta_{(y, z)} \mu\right)(w) \mathrm{d} t \mathrm{~d} w .
$$

Collecting (10)-(12), we deduce from (9) that

$\int_{Q_{T}} u^{2} \mu \mathrm{d} t \mathrm{~d} w \leq c_{T}\left(\int_{Q_{R, T}}\left|u\left(\Delta_{(y, z)} \mu\right)\right| \mathrm{d} t \mathrm{~d} w+\int_{Q_{R, T}}\left|u \partial_{x} \mu\right| \mathrm{d} t \mathrm{~d} w+\int_{B_{T}}\left|u_{0} \partial_{x} \mu\right| \mathrm{d} w\right)+\int_{\mathbb{R}^{3}}\left(T u_{0}^{2}+T^{2} u_{0} u_{1}\right) \mu \mathrm{d} w$.

Applying $\varepsilon$-Young inequality, we get 


$$
\int_{Q_{R, T}}|u|\left(\left|\Delta_{(y, z)} \mu\right|+\left|\partial_{x} \mu\right|\right) \mathrm{d} t \mathrm{~d} w \leq \varepsilon \int_{Q_{R, T}} u^{2} \mu \mathrm{d} t \mathrm{~d} w+c_{\varepsilon} \int_{B_{R}} \frac{\left|\Delta_{(y, z)} \mu\right|^{2}+\left|\partial_{x} \mu\right|^{2}}{\mu} \mathrm{d} w .
$$

Using this and Lemma 2.2, (13) becomes

$$
\int_{Q_{T}} u^{2} \mu \mathrm{d} t \mathrm{~d} w \leq c_{T}\left(1+\int_{B_{T}}\left|u_{0} \partial_{x} \mu\right| \mathrm{d} w\right)+\int_{\mathbb{R}^{3}}\left(T u_{0}^{2}+T^{2} u_{0} u_{1}\right) \mu \mathrm{d} w .
$$

Since $u_{0}^{2}, u_{0} u_{1} \in L^{1}\left(\mathbb{R}^{3}\right)$, then $\int_{B_{T}}\left|u_{0} \partial_{x} \mu\right| \mathrm{d} w$ tends to zero as $R$ tends to $+\infty$. This yields

$$
\int_{Q_{T}} u^{2} \mu \mathrm{d} t \mathrm{~d} w \leq c_{T}+\int_{\mathbb{R}^{3}}\left(T u_{0}^{2}+T^{2} u_{0} u_{1}\right) \mathrm{d} w .
$$

Hence $u$ belongs to $L^{2}\left(\mathbb{R}^{3}\right)$.

Now, applying Hölder to (13), we obtain

$$
\int_{Q_{T}} u^{2} \mu \mathrm{d} t \mathrm{~d} w \leq c_{T}\left(\int_{Q_{R, T}} u^{2} \mu \mathrm{d} t \mathrm{~d} w\right)^{\frac{1}{2}}\left(\int_{B_{R}} \frac{\left|\Delta_{(y, z)} \mu\right|^{2}+\left|\partial_{x} \mu\right|^{2}}{\mu} \mathrm{d} w\right)^{\frac{1}{2}}+\int_{B_{T}}\left|u_{0} \partial_{x} \mu\right| \mathrm{d} w+\int_{\mathbb{R}^{3}}\left(T u_{0}^{2}+T^{2} u_{0} u_{1}\right) \mu \mathrm{d} w .
$$

Finally, since $u \in L^{2}\left(Q_{T}\right)$, then $\int_{Q_{R, T}} u^{2} \mu \mathrm{d} t \mathrm{~d} w$ tends to zero as $R$ tends to $+\infty$. This yields

$$
\int_{Q_{T}} u^{2}(t, w) \mathrm{d} t \mathrm{~d} w \leq \int_{\mathbb{R}^{3}}\left(T u_{0}^{2}(w)+T^{2} u_{0}(w) u_{1}(w)\right) \mathrm{d} w
$$

Then the result of Theorem 3.1 holds.

Assume that there exists a pair $(u, v)$ solution to system (3)-(4), defined on $Q_{T}$ for some $T>0$. Then

$$
\frac{1}{2} \int_{Q_{T}} \eta\left(\partial_{t}^{\alpha+1} v^{2}\right) \mathrm{d} t \mathrm{~d} w=\int_{Q_{T}} \eta\left(\partial_{t}^{\alpha} \partial_{x} u\right) \mathrm{d} t \mathrm{~d} w-\int_{Q_{T}} \eta\left(\Delta_{(y, z)} u\right) \mathrm{d} t \mathrm{~d} w
$$

and

$$
\frac{1}{2} \int_{Q_{T}} \eta\left(\partial_{t}^{\beta+1} u^{2}\right) \mathrm{d} t \mathrm{~d} w=\int_{Q_{T}} \eta\left(\partial_{t}^{\beta} \partial_{x} v\right) \mathrm{d} t \mathrm{~d} w-\int_{Q_{T}} \eta\left(\Delta_{(y, z)} v\right) \mathrm{d} t \mathrm{~d} w
$$

The analogue of the estimation (13), for system (3)-(4), is the following

$$
\int_{Q_{T}} v^{2} \mu \mathrm{d} t \mathrm{~d} w \leq c_{T}\left(\int_{Q_{R, T}}\left|u\left(\Delta_{(y, z)} \mu\right)\right| \mathrm{d} t \mathrm{~d} w+\int_{Q_{R, T}}\left|u \partial_{x} \mu\right| \mathrm{d} t \mathrm{~d} w+\int_{B_{T}}\left|u_{0} \partial_{x} \mu\right| \mathrm{d} w\right)+\int_{\mathbb{R}^{3}}\left(T v_{0}^{2}+T^{2} v_{0} v_{1}\right) \mu \mathrm{d} w
$$

together with

$$
\int_{Q_{T}} u^{2} \mu \mathrm{d} t \mathrm{~d} w \leq c_{T}\left(\int_{Q_{R, T}}\left|v\left(\Delta_{(y, z)} \mu\right)\right| \mathrm{d} t \mathrm{~d} w+\int_{Q_{R, T}}\left|v \partial_{x} \mu\right| \mathrm{d} t \mathrm{~d} w+\int_{B_{T}}\left|v_{0} \partial_{x} \mu\right| \mathrm{d} w\right)+\int_{\mathbb{R}^{3}}\left(T u_{0}^{2}+T^{2} u_{0} u_{1}\right) \mu \mathrm{d} w .
$$

As in the case of single equation, we use $\varepsilon$-Young and Hölder inequalities to deduce that

$$
\int_{Q_{T}} u^{2} \mathrm{~d} t \mathrm{~d} w \leq \int_{\mathbb{R}^{3}}\left(T u_{0}^{2}+T^{2} u_{0} u_{1}\right) \mathrm{d} w \text { and } \int_{Q_{T}} v^{2} \mathrm{~d} t \mathrm{~d} w \leq \int_{\mathbb{R}^{3}}\left(T v_{0}^{2}+T^{2} u_{0} v_{1}\right) \mathrm{d} w .
$$

Therefore, the result in Theorem 3.2 holds. 
Acknowledgements: This work was supported by Researchers Supporting Project number (RSP-2020/57), King Saud University, Riyadh, Saudi Arabia.

\section{References}

[1] Mohamed Jleli, Instantaneous blow-up for a fractional-in-time evolution equation arising in plasma theory, Math. Methods Appl. Sci. (2020), DOI: 10.1002/mma.6309.

[2] Maxim O. Korpusov, Aleksandra K. Matveeva, and Dmitry V. Lukyanenko, Diagnostics of instant decomposition of solution in the nonlinear equation of theory of waves in semiconductors, Vestnik YuUrGU. Ser. Mat. Model. Progr. 12 (2019), no. 4, 104-113, DOI: 10.14529/mmp190408.

[3] Maxim O. Korpusov and Evgenii A. Ovsyannikov, Blow-up instability in non-linear wave models with distributed parameters, Izv. Math. 84 (2020), no. 3, 449-501, DOI: 10.1070/IM8820.

[4] Sergey N. Gurbatov, Oleg V. Rudenko, and Aleksandr I. Saichev, Waves and Structures in Nonlinear Nondispersive Media, Springer-Verlag Berlin Heidelberg, 2011.

[5] Maxim O. Korpusov and S. Mikhailenko, Instantaneous blow-up of classical solutions to the Cauchy problem for the Khokhlov-Zabolotskaya equation, Comput. Math. Math. Phys. 57 (2017), no. 7, 1167-1172, DOI: 10.1134/ S0965542517030095.

[6] Enzo Mitidieri and Stanislav I. Pohozaev, Apriori estimates and blow-up of solutions to partial differential equations and inequalities, Proc. Steklov Inst. Math. 234 (2001), 3-383.

[7] Stefan G. Samko, Anatoly A. Kilbas, and Oleg I. Marichev, Fractional Integrals and Derivatives: Theory and Applications, Gordon and Breach Science Publishers, Yverdon, Switzerland, 1993. 